\title{
Equivariant Riemann-Roch theorems for curves over perfect fields
}

Helena Fischbacher-Weitz

Department of Pure Mathematics

University of Sheffield

Hicks Building, Hounsfield Road

Sheffield

S3 7RH

h.fischbacher-weitz@shef.ac.uk
Bernhard Köck (communicating author)

School of Mathematics

University of Southampton

Highfield

Southampton

SO17 1BJ

b.koeck@soton.ac.uk

Tel. 00442380595125

Fax 00442380595147

\begin{abstract}
We prove an equivariant Riemann-Roch formula for divisors on algebraic curves over perfect fields. By reduction to the known case of curves over algebraically closed fields, we first show a preliminary formula with coefficients in $\mathbb{Q}$. We then prove and shed some further light on a divisibility result that yields a formula with integral coefficients. Moreover, we give variants of the main theorem for equivariant locally free sheaves of higher rank.
\end{abstract}




\section{Introduction}

Let $X$ be a smooth, projective, geometrically irreducible curve over a perfect field $\mathrm{k}$ and let $G$ be a finite subgroup of the automorphism group $\operatorname{Aut}(X / k)$. For any locally free $G$-sheaf $\mathcal{E}$ on $X$, we are interested in computing the equivariant Euler characteristic

$$
\chi(G, X, \mathcal{E}):=\left[H^{0}(X, \mathcal{E})\right]-\left[H^{1}(X, \mathcal{E})\right] \in K_{0}(G, k),
$$

considered as an element of the Grothendieck group $K_{0}(G, k)$ of finitely generated modules over the group ring $k[G]$. The main example of a locally free $G$-sheaf we have in mind is the sheaf $\mathcal{L}(D)$ associated with a $G$-equivariant divisor $D=\sum_{P \in X} n_{P} P$ (that is $n_{\sigma(P)}=n_{P}$ for all $\sigma \in G$ and all $\left.P \in X\right)$. If two $k[G]$-modules are in the same class in $K_{0}(G, k)$, they are not necessarily isomorphic when the characteristic of $k$ divides the order of $G$. In order to be able to determine the actual $k[G]$-isomorphism class of $H^{0}(X, \mathcal{E})$ or $H^{1}(X, \mathcal{E})$, we are therefore also interested in deriving conditions for $\chi(G, X, \mathcal{E})$ to lie in the Grothendieck group $K_{0}(k[G])$ of finitely generated projective $k[G]$-modules and in computing $\chi(G, X, \mathcal{E})$ within $K_{0}(k[G])$.

The equivariant Riemann-Roch problem goes back to Chevalley and Weil [CW], who described the $G$-structure of the space of global holomorphic differentials on a compact Riemann surface. Ellingsrud and Lønsted [EL] found a formula for the equivariant Euler characteristic of an arbitrary $G$-sheaf on a curve over an algebraically closed field of characteristic zero. Nakajima $[\mathrm{Na}]$ and Kani $[\mathrm{Ka}]$ independently generalized this to curves over arbitrary algebraically closed fields, under the assumption that the canonical morphism $X \rightarrow X / G$ be tamely ramified. These results have been revisited by Borne [Bo], who also found a formula that computes the difference between the equivariant Euler characteristics of two $G$-sheaves in the case of a wildly ramified cover $X \rightarrow X / G$. In the same setting, formulae for the equivariant Euler characteristic of a single $G$-sheaf have been found by the second author ([Kö1], [Kö2]). Using these formulae, new proofs for the reults of Ellingsrud-Lønsted, Nakajima and Kani have been given [Kö1].

In this paper, we concentrate on the case where the underlying field $k$ is perfect. Our main theorem, Theorem 3.4, is an equivariant Riemann-Roch formula in $K_{0}(k[G])$ when the canonical morphism $X \rightarrow X / G$ is weakly ramified and $\mathcal{E}=\mathcal{L}(D)$ for some equivariant divisor $D$. By reduction to the known case of curves over algebraically closed fields, we first show a preliminary formula with coefficients in $\mathbb{Q}$. The divisibility result needed to obtain a formula with integral coefficients is then proved in two ways: Firstly, by applying the preliminary formula to suitably chosen equivariant divisors; and secondly, in two situations, by a local argument. The following paragraphs describe the content of each section in more detail.

It is well-known that a finitely generated $k[G]$-module $M$ is projective if and only if $M \otimes_{k} \bar{k}$ is a projective $\bar{k}[G]$-module. In Section 2 we give a variant of this fact for classes in $K_{0}(G, k)$ rather than for $k[G]$-modules $M$ (Corollary 2.2). This variant is much harder to prove and is an essential tool for the proof of our main result in Section 3.

The first results in Section 3 give both a sufficient condition and a necessary condition under which the equivariant Euler characteristic $\chi(G, X, \mathcal{E})$ lies in the image of the Cartan homomorphism $c: K_{0}(G, k) \rightarrow K_{0}(k[G])$. More precisely, when $\mathcal{E}=\mathcal{L}(D)$ for some equivariant divisor $D=\sum_{P \in X} n_{P} P$, this holds if the canonical projection $\pi: X \rightarrow X / G$ is weakly ramified and $n_{P}+1$ is divisible by the wild part $e_{P}^{w}$ of the ramification index $e_{P}$ for all $P \in X$. When $\pi$ is weakly ramified we furthermore derive from the corresponding result in [Kö2] the existence of the so-called ramification module $N_{G, X}$, a certain projective $k[G]$-module which embodies a global relation between the (local) representations 
$\mathfrak{m}_{P} / \mathfrak{m}_{P}^{2}$ of the inertia group $I_{P}$ for $P \in X$. If moreover $D$ is an equivariant divisor as above, our main result, Theorem 3.4, expresses $\chi(G, X, \mathcal{L}(D))$ as an integral linear combination in $K_{0}(k[G])$ of the classes of $N_{G, X}$, the regular representation $k[G]$ and the projective $k[G]$-modules $\operatorname{Ind}_{G_{P}}^{G}\left(W_{P, d}\right)$ (for $P \in X$ and $d \geq 0$ ) where the projective $k\left[G_{P}\right]$-module $W_{P, d}$ is defined by the following isomorphism of $k\left[G_{P}\right]$-modules:

$$
\operatorname{Ind}_{I_{P}}^{G_{P}}\left(\operatorname{Cov}\left(\left(\mathfrak{m}_{P} / \mathfrak{m}_{P}^{2}\right)^{\otimes(-d)}\right)\right) \cong \bigoplus^{f_{P}} W_{P, d}
$$

here Cov means taking the $k\left[I_{P}\right]$-projective cover and $f_{P}$ denotes the residual degree.

Finding an equivariant Riemann-Roch formula without denominators amounts to showing that $W_{P, d}$ exists, i.e. that the left-hand side of the above is "divisible by $f_{P}$ ". To do this, we use our prototype formula with denominators, formula (4), and apply it to certain equivariant divisors $D$. If $\pi$ is tamely ramified, we furthermore consider two situations where we can give a local proof of the divisibility result, yielding a more concrete description of $W_{P, d}$, see Proposition 3.5.

In Section 4, we give some variants of the main result that hold under slightly different assumptions. In particular, these variants hold for locally free $G$-sheaves that do not necessarily come from a divisor.

\section{Preliminaries}

The purpose of this section is to fix some notations used throughout this paper and to state some folklore results used later.

Throughout this section, let $X$ be a scheme of finite type over a field $k$, and let $\bar{k}$ be an algebraic closure of $k$. For any (closed) point $P \in X$, let $k(P):=\mathcal{O}_{X, P} / \mathfrak{m}_{P}$ denote the residue field at $P$. Throughout this paper, let $\bar{X}$ denote the geometric fibre $X \times_{k} \bar{k}$, which is a scheme of finite type over $\bar{k}$, and let $p$ denote the canonical projection $\bar{X} \rightarrow X$. Recall that $p$ is a closed, flat morphism which is in general not of finite type. We will see later that in dimension $1, p$ is "unramified" in the sense that if $Q \in \bar{X}$ and $P=p(Q)$, then a local parameter at $P$ is also a local parameter at $Q$. By Galois theory and Hilbert's Nullstellensatz, we have for every $P \in \bar{X}$ :

$$
\# p^{-1}(P)=\# \operatorname{Hom}_{k}(k(P), \bar{k}) \leq[k(P): k]<\infty,
$$

and equality holds if $k(P) / k$ is separable.

Let now $G$ be a finite subgroup of $\operatorname{Aut}(X / k)$. Since the homomorphism

$$
\operatorname{Aut}(X / k) \rightarrow \operatorname{Aut}(\bar{X} / \bar{k}), \sigma \mapsto \sigma \times \text { id }
$$

is injective, which is easy to check, we may view $G$ as a subgroup of $\operatorname{Aut}(\bar{X} / \bar{k})$. Since the elements of $G$ act on the topological space of $X$ as homeomorphisms, $G$ also acts on $|X|$, the set of closed points in $X$. Analogously, $G$ acts on the set $|\bar{X}|$ of closed points in $\bar{X}$.

Definition 1.1. A locally free $G$-sheaf (of rank $r$ ) on $X$ is a locally free $\mathcal{O}_{X}$-module $\mathcal{E}$ (of rank $r$ ) together with an isomorphism of $\mathcal{O}_{X}$-modules $v_{\sigma}: \sigma^{*} \mathcal{E} \rightarrow \mathcal{E}$ for every $\sigma \in G$, such that for all $\sigma, \tau \in G$, the following diagram commutes:

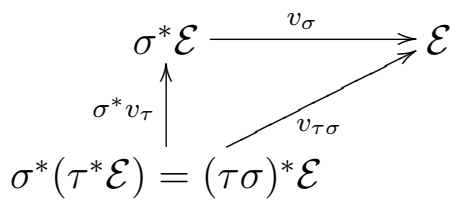


If $\mathcal{E}$ is a locally free $G$-sheaf of finite rank, then the cohomology groups $H^{i}(X, \mathcal{E})\left(i \in \mathbb{N}_{0}\right)$ are $k$-representations of $G$. If moreover $X$ is proper over $k$, then the $H^{i}(X, \mathcal{E})$ are finitedimensional and vanish for $i>>0$ (see Theorem III.5.2 in [Ha]).

We denote the Grothendieck group of all finitely generated $k[G]$-modules (i.e. finitedimensional $k$-representations of $G$ ) by $K_{0}(G, k)$, as opposed to the notation $R_{k}(G)$ used by Serre in [Se2].

Definition 1.2. If $X$ is proper over $k$, and $\mathcal{E}$ is a locally free $G$-sheaf of finite rank, then

$$
\chi(G, X, \mathcal{E}):=\sum_{i}(-1)^{i}\left[H^{i}(X, \mathcal{E})\right] \in K_{0}(G, k)
$$

is called the equivariant Euler characteristic of $\mathcal{E}$ on $X$.

For $P \in|X|$ or $P \in|\bar{X}|$, the decomposition group $G_{P}$ and the inertia group $I_{P}$ are defined as follows:

$$
\begin{aligned}
G_{P} & :=\{\sigma \in G \mid \sigma(P)=P\} \\
I_{P} & :=\left\{\sigma \in G_{P} \mid \bar{\sigma}=\operatorname{id}_{k(P)}\right\}=\operatorname{ker}\left(G_{P} \rightarrow \operatorname{Aut}(k(P) / k)\right) .
\end{aligned}
$$

Here $\bar{\sigma}$ denotes the endomorphism that $\sigma$ induces on $k(P)$. Note that for all $Q \in|\bar{X}|$, we have $G_{Q}=I_{Q}$ and $G_{Q}=I_{P}$, where $P:=p(Q) \in|X|$.

In the following lemma, we will assume for the first time that the field $k$ is perfect.

Lemma 1.3. Assume that $k$ is perfect. Let $\mathcal{F}$ be a coherent sheaf on $X$, and let $\overline{\mathcal{F}}:=$ $p^{*} \mathcal{F}$. Let $P$ be a point in $X$, and let $\mathcal{F}(P):=\mathcal{F}_{P} \otimes_{\mathcal{O}_{X, P}} k(P)$ be the fibre of $\mathcal{F}$ at $P$. Then the canonical homomorphism

$$
\mathcal{F}(P) \otimes_{k} \bar{k} \mapsto \bigoplus_{Q \in p^{-1}(P)} \overline{\mathcal{F}}(Q)
$$

is an isomorphism. In particular, the canonical homomorphism

$$
k(P) \otimes_{k} \bar{k} \rightarrow \bigoplus_{Q \in p^{-1}(P)} k(Q)
$$

is an isomorphism.

Proof. It follows from Galois theory that for any separable finite field extension $k^{\prime} / k$, the homomorphism

$$
k^{\prime} \otimes_{k} \bar{k} \rightarrow \bigoplus_{\operatorname{Hom}_{k}\left(k^{\prime}, \bar{k}\right)} \bar{k}
$$

defined by

$$
y \otimes z \mapsto(\varphi(y) \cdot z)_{\varphi \in \operatorname{Hom}_{k}\left(k^{\prime}, \bar{k}\right)}
$$

is an isomorphism. Since $k$ is perfect, by putting $k^{\prime}=k(P)$ this implies the second part of the lemma, i.e. the special case where $\mathcal{F}=\mathcal{O}_{X}$.

Since the lemma is a local statement on $X$, we may assume that $X$ is affine. The general case then follows from the special case together with the definitions and basic properties of coherent sheaves and fibred products. 
Proposition 1.4. Assume that $k$ is perfect. Let $\Omega_{X / k}$ be the sheaf of relative differentials of $X$ over $k$. Then for every point $P \in|X|$, the canonical map

$$
\mathfrak{m}_{P} / \mathfrak{m}_{P}^{2} \rightarrow \Omega_{X / k}(P)
$$

is an isomorphism.

Proof. Let $\Omega_{k(P) / k}$ denote the module of relative differential forms of $k(P)$ over $k$. Using some basic properties of differentials and of the cotangent space in an affine setting, it follows from Corollary 6.5 in $[\mathrm{Ku}]$ that we have an exact sequence

$$
0 \rightarrow \mathfrak{m}_{P} / \mathfrak{m}_{P}^{2} \rightarrow \Omega_{X / k}(P) \rightarrow \Omega_{k(P) / k} \rightarrow 0 .
$$

By Corollary 5.3 in $[\mathrm{Ku}], \Omega_{k(P) / k}$ is trivial, so the map $\mathfrak{m}_{P} / \mathfrak{m}_{P}^{2} \rightarrow \Omega_{X / k}(P)$ is an isomorphism.

Note that both Corollary 6.5 and Corollary 5.3 in $[\mathrm{Ku}]$ require $k(P) / k$ to be separable.

Both Lemma 1.3 and Proposition 1.4 can be turned into equivariant statements in the following sense. If we require $\mathcal{F}$ to be a locally free $G$-sheaf, then for every point $P \in|X|$, we obtain an action of the inertia group $I_{P}$ on the fibre $\mathcal{F}(P)$ by $k(P)$-automorphisms. The action of $I_{P}$ on the fibre $\Omega_{X}(P)$ of the canonical sheaf corresponds to the action on the cotangent space $\mathfrak{m}_{P} / \mathfrak{m}_{P}^{2}$ via the isomorphism from Proposition 1.4.

By letting $I_{P}$ act trivially on $\bar{k}$, we can extend the action of $I_{P}$ on $\mathcal{F}(P)$ to an action on the tensor product $\mathcal{F}(P) \otimes_{k} \bar{k}$. On the other hand, since $I_{Q}=I_{P}$ for any point $Q \in p^{-1}(P), I_{P}$ acts on the fibre $\mathcal{G}(Q)$ of any locally free $G$-sheaf $\mathcal{G}$ on $\bar{X}$ for any point $Q \in p^{-1}(P)$. In particular, this holds if $\mathcal{G}=p^{*} \mathcal{F}$ for a locally free $G$-sheaf $\mathcal{F}$ on $X$. With respect to these group actions, the isomorphism from Lemma 1.3 is an isomorphism of $\bar{k}\left[I_{P}\right]$-modules.

We also have an action of the decomposition group $G_{P}$ on any fibre $\mathcal{F}(P)$, but $G_{P}$ only acts on the fibre via $k$-automorphisms, whereas $I_{P}$ acts via $k(P)$-automorphisms. $G_{P}$ does act $k(P)$-semilinearly on the fibre, that is, for any $\sigma \in G_{P}, a \in k(P)$ and $x, y \in \mathcal{F}(P)$ we have $\sigma \cdot(a x+y)=(\bar{\sigma} . a)(\sigma \cdot x)+\sigma \cdot y$, where $\bar{\sigma}$ denotes the automorphism of $k(P) / k$ induced by $\sigma$.

Let now $X$ be a smooth, projective curve over a perfect field k. Assume further that $X$ is geometrically irreducible, i.e. that the geometric fibre $\bar{X}=X \times_{k} \bar{k}$ is irreducible. Then the curve $X$ itself is irreducible.

The following lemma shows that although the canonical morphism $p: \bar{X} \rightarrow X$ is usually not of finite type, it can be thought of as an "unramified" morphism in the common sense, a fact that will be used frequently throughout this paper.

Lemma 1.5. Let $Q \in|\bar{X}|$ be a closed point, and let $P:=p(Q)$. Then every local parameter at $P$ is also a local parameter at $Q$.

Proof. Let $t_{P}$ be a local parameter at $P$. Then $t_{P}$ must be an element of $\mathfrak{m}_{P} \backslash \mathfrak{m}_{P}^{2}$, so (the equivalence class of) $t_{P}$ is a generator of the one-dimensional vector space $\mathfrak{m}_{P} / \mathfrak{m}_{P}^{2}$ over $k(P)$. Hence, $t_{P} \otimes 1$ is a generator of the rank-1 module $\mathfrak{m}_{P} / \mathfrak{m}_{P}^{2} \otimes_{k} \bar{k}$ over $k(P) \otimes_{k} \bar{k}$. By Lemma 1.3 and Proposition 1.4, we have a canonical isomorphism

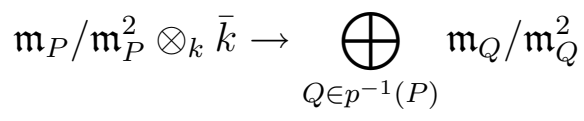


which we can view as an isomorphism of modules over $k(P) \otimes_{k} \bar{k} \cong \bigoplus_{Q \in p^{-1}(P)} k(Q)$. Since this isomorphism must map $t_{P} \otimes 1$ to a generator of the right-hand side over $\bigoplus_{Q \in p^{-1}(P)} k(Q)$, the image of $t_{P} \otimes 1$ in each component $\mathfrak{m}_{Q} / \mathfrak{m}_{Q}^{2}$ must be a generator of $\mathfrak{m}_{Q} / \mathfrak{m}_{Q}^{2}$, i.e. the image of $t_{P}$ under each induced homomorphism $p_{Q}: \mathcal{O}_{X, P} \rightarrow \mathcal{O}_{\bar{X}, Q}$ must be a local parameter at $Q$.

Let now $G$ be a finite subgroup of $\operatorname{Aut}(X / k)$. It is a well-known result that the quotient scheme $Y:=X / G$ is also a smooth projective curve, with function field $K(Y)=K(X)^{G}$. The canonical projection $X \rightarrow Y$ will be called $\pi$. Let $P \in X$ be a closed point, $R:=\pi(P) \in Y$. Let $v_{p}$ be the unique normed valuation of the function field $K(X)$ associated to $P$, and let $v_{R}$ be the unique normed valuation of $K(Y)$ associated to $R$. Then $v_{P}$ is equivalent to a valuation extending $v_{R}$. For $s \geq-1$, we define the $s$-th ramification group $G_{P, s}$ at $P$ to be the $s$-th ramification group of the extension of local fields $K(X)_{v_{P}} / K(Y)_{v_{R}}$. In particular, we have $G_{P,-1}=G_{P}$ and $G_{P, 0}=I_{P}$.

The canonical projection $\pi: X \rightarrow Y$ is called unramified (tamely ramified, weakly ramified) if $G_{P, s}$ is trivial for $s \geq 0(s \geq 1, s \geq 2)$ and for all $P \in X$. We denote the ramification index of $\pi$ at the place $P$ by $e_{P}$, its wild part by $e_{P}^{w}$ and its tame part by $e_{P}^{t}$. In other words, $e_{P}=v_{P}\left(t_{\pi(P)}\right)=\left|G_{P, 0}\right|, e_{P}^{w}=\left|G_{P, 1}\right|$ and $e_{P}^{t}=\left|G_{P, 0} / G_{P, 1}\right|$.

If $Q \in|\bar{X}|$ is a closed point, $P:=p(Q) \in|X|$, then for every $s \geq 0$, we have $G_{Q, s}=G_{P, s}$ (by Proposition 5 in Chapter IV in [Se1] and Lemma 1.5). In particular, we have $e_{P}=e_{Q}$, $e_{P}^{w}=e_{Q}^{w}$ and $e_{P}^{t}=e_{Q}^{t}$.

\section{A Cartesian diagram of Grothendieck groups}

A $k[G]$-module $M$ is projective if and only if $M \otimes_{k} \bar{k}$ is a projective $\bar{k}[G]$-module. In this section, we will now show variants of this well-known fact for classes in $K_{0}(G, k)$ rather than $k[G]$-modules.

Let $K_{0}(k[G])$ denote the Grothendieck group of finitely generated projective $k[G]$ modules. This is a free group generated by the isomorphism classes of indecomposable projective $k[G]$-modules. The Cartan homomorphisms $c: K_{0}(k[G]) \rightarrow K_{0}(G, k)$ and $\bar{c}: K_{0}(\bar{k}[G]) \rightarrow K_{0}(G, \bar{k})$ are injective ([Se2], 16.1, Corollary 1 of Theorem 35), so $K_{0}(k[G])$ may be viewed as a subgroup of $K_{0}(G, k)$. The homomorphism

$$
\beta: K_{0}(G, k) \rightarrow K_{0}(G, \bar{k})
$$

defined by tensoring with $\bar{k}$ over $k$ restricts to a homomorphism

$$
\alpha: K_{0}(k[G]) \rightarrow K_{0}(\bar{k}[G]) .
$$

By Proposition (16.22) in [CR], both homomorphisms $\beta, \alpha$ are split injections.

Proposition 2.1. The following diagram with injective arrows is Cartesian, i.e. it commutes and viewing the injections as inclusions, we have $K_{0}(\bar{k}[G]) \cap K_{0}(G, k)=K_{0}(k[G])$.

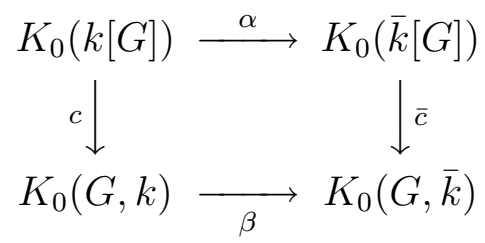


Proof. The commutativity is obvious. Now consider the extended diagram (with exact rows)

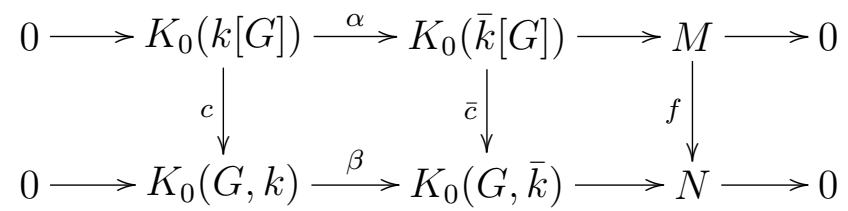

where $M=\operatorname{cok} \alpha, N=\operatorname{cok} \beta$, and $f$ is the homomorphism $M \rightarrow N$ induced by $\bar{c}$. By the Snake Lemma, there is an exact sequence of abelian groups

$$
0 \rightarrow \operatorname{ker} c \rightarrow \operatorname{ker} \bar{c} \rightarrow \operatorname{ker} f \rightarrow \operatorname{cok} c,
$$

the first two modules being trivial since $c$ and $\bar{c}$ are injective. Since $\alpha$ is a split injection, $M=\operatorname{cok} \alpha$ is free over $\mathbb{Z}$, and therefore ker $f$ must also be free over $\mathbb{Z}$. On the other hand, by Theorem (21.22) in [CR], we have $|G| \cdot \operatorname{cok} c=0$, so $\operatorname{cok} c$ is a torsion module. Using the exactness of the sequence above, this implies ker $f=0$. Now an easy diagram chase completes the proof.

Proposition 2.1 says that given a class $\mathcal{C}$ in $K_{0}(G, k), \mathcal{C}$ lies in the image of $c$ if and only if $\beta(\mathcal{C})$ lies in the image of $\bar{c}$. The following corollary appears to be only slightly different from this, yet some additional tools will be required for its proof.

Corollary 2.2. Let $\mathcal{C}$ be a class in $K_{0}(G, k)$. Then $\mathcal{C}$ is the class of a projective $k[G]-$ module if and only if $\beta(\mathcal{C})$ is the class of a projective $\bar{k}[G]$-module.

Before proving Corollary 2.2, we will need a few preliminary results on $k[G]$-modules. Recall that a $k[G]$-module is called simple if it is nonzero and has no proper $k[G]$ submodules, and indecomposable if it is nonzero and is not a direct sum of proper $k[G]$ submodules.

Proposition 2.3. (a) For every simple $k[G]$-module $M$, the $\bar{k}[G]$-module $M \otimes_{k} \bar{k}$ is semisimple.

(b) Let $\left\{P_{1}, \ldots, P_{s}\right\}$ be a set of representatives of the isomorphism classes of indecomposable projective $k[G]$-modules, and let

$$
P_{i} \otimes_{k} \bar{k}=\bigoplus_{j=1}^{r_{i}} \bar{Q}_{i j}, \quad \bar{Q}_{i j} \text { indecomposable projective } \bar{k}[G] \text {-modules. }
$$

Then every indecomposable $\bar{k}[G]$-module is isomorphic to some $\bar{Q}_{i j}$. Further $\bar{Q}_{i j} \cong \bar{Q}_{i^{\prime} j^{\prime}}$ implies that $i=i^{\prime}$, i.e. there is no overlap between the sets of indecomposable $\bar{k}[G]$-modules which come from different indecomposable $k[G]$-modules.

Proof. This proposition is a variation of Theorem 7.9 in [CR]. In [CR], the algebraic closure $\bar{k}$ is replaced by a finite algebraic extension $E$ of $k$, and part (b) is stated for simple modules rather than for indecomposable projective modules. Using only elementary algebraic methods, it can be shown that there is a finite algebraic extension $E / k$ such that every simple $\bar{k}[G]$-module can be realized as a simple $E[G]$-module, i.e. every simple $\bar{k}[G]$-module $M$ can be written as $M=N \otimes_{E} \bar{k}$ for some simple $E[G]$-module $N$. This suffices to derive part (a) from the result in [CR]. Furthermore, it is well-known that mapping every projective $k[G]$-module $P$ to the $k[G]$-module $P / \operatorname{rad} P$ gives a $1-1$ correspondence between the isomorphism classes of indecomposable projective $k[G]$-modules 
and the isomorphism classes of simple $k[G]$-modules, whose inverse is given by taking $k[G]$-projective covers. We can thus deduce our proposition from the result in [CR], using that projective covers are additive (by Corollary 6.25 (ii) in [CR]) and commute with tensor products (by Corollary 6.25 (i) in [CR]).

Proof of Corollary 2.2. The "only if" direction is obvious. For the "if" direction, we note first of all that if $\mathcal{C}$ is a class in $K_{0}(G, k)$ and $\beta(\mathcal{C})$ is the class of a projective $\bar{k}[G]$ module, then Proposition 2.1 yields that $\mathcal{C}$ can be viewed as a class in $K_{0}(k[G])$. Hence it suffices to show the "if" direction for classes $\mathcal{C} \in K_{0}(k[G])$, replacing the homomorphism $\beta$ by its restriction $\alpha$.

Let $\left\{P_{1}, \ldots, P_{s}\right\}$ be a set of representatives of the isomorphism classes of indecomposable $k[G]$-modules. Every $\mathcal{C} \in K_{0}(k[G])$ can now be written as a $\mathbb{Z}$-linear combination of the classes $\left[P_{i}\right]$, and all coefficients of this linear combination are nonnegative if and only if $\mathcal{C}$ is the class of a projective module. Using Proposition 2.3, one now easily shows that if $\alpha(\mathcal{C})$ is the class of a projective module in $K_{0}(\bar{k}[G])$, then $\mathcal{C}$ is the class of a projective module in $K_{0}(k[G])$, which proves the assertion.

\section{The equivariant Euler characteristic in terms of projective $k[G]$-modules}

By a theorem of Nakajima, the equivariant Euler characteristic of any locally free $G$-sheaf on $X$ lies in the image of the Cartan homomorphism $c: K_{0}(k[G]) \rightarrow K_{0}(G, k)$, provided that the canonical projection $\pi: X \rightarrow Y=X / G$ is tamely ramified. In this section, we will also consider the more general case where $\pi$ is weakly ramified. We give both a necessary condition and a sufficient condition for the equivariant Euler characteristic to lie in the image of $c$, provided that the $G$-sheaf in question has rank 1 (comes from a divisor). Under this condition, we state an equivariant Riemann-Roch formula in the Grothendieck group of projective $k[G]$-modules.

We make the same assumptions and use the same notations as in section 1 . In particular $p$ denotes the projection $\bar{X}=X \times_{k} \bar{k} \rightarrow X$. Additionally, let $\bar{\pi}$ denote the canonical projection $\bar{X} \rightarrow \bar{Y}:=\bar{X} / G=Y \otimes_{k} \bar{k}$, and let $\tilde{p}$ denote the projection $\bar{Y} \rightarrow Y$. We have the following commutative diagram:

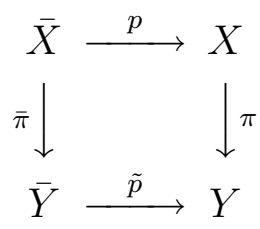

Theorem 3.1. If $\pi$ is tamely ramified and $\mathcal{E}$ is a locally free $G$-sheaf on $X$, then the equivariant Euler characteristic $\chi(G, X, \mathcal{E})$ lies in the image of the Cartan homomorphism $c: K_{0}(k[G]) \rightarrow K_{0}(G, k)$.

Proof. Follows directly from Theorem 1 in $[\mathrm{Na}]$.

Theorem 3.2. Let $D=\sum_{P \in|X|} n_{P} P$ be a $G$-equivariant divisor on $X$.

(a) If $\pi$ is weakly ramified and $n_{P} \equiv-1 \bmod e_{P}^{w}$ for all $P \in X$, then the equivariant Euler characteristic $\chi(G, X, \mathcal{L}(D))$ lies in the image of the Cartan homomorphism $c: K_{0}(k[G]) \rightarrow K_{0}(G, k)$. If moreover one of the cohomology groups $H^{i}(X, \mathcal{L}(D))$, $i=0,1$, vanishes, then the other one is a projective $k[G]$-module. 
(b) Let $\operatorname{deg} D>2 g_{X}-2$. If the $k[G]$-module $H^{0}(X, \mathcal{L}(D))$ is projective, then $\pi$ is weakly ramified and $n_{P} \equiv-1 \bmod e_{P}^{w}$ for all $P \in|X|$.

Proof. If $k$ is algebraically closed, the theorem coincides with Theorem 2.1 in [Kö2].

In the general case, if $\pi$ is weakly ramified and $D$ satisfies the congruence condition " $n_{P} \equiv-1 \bmod e_{P}^{w}$ for all $P$ ", then $\bar{\pi}: \bar{X} \rightarrow \bar{Y}$ is weakly ramified, and by Lemma 1.5, the divisor $p^{*} D$ on $\bar{X}$ also satisfies the congruence condition. By the special case, $\chi\left(G, X, \mathcal{L}\left(p^{*} D\right)\right.$ then lies in the image of $\bar{c}$. Hence by Proposition $2.1, \chi(G, X, \mathcal{L}(D)$ lies in the image of $c$. Here we have used that $H^{i}(X, \mathcal{L}(D)) \otimes_{k} \bar{k}=H^{i}\left(\bar{X}, \mathcal{L}\left(p^{*} D\right)\right)$ for every $i$ (cf. Proposition III.9.3 in [Ha]). This also implies the rest of part (a).

For part (b), let $\operatorname{deg} D>2 g_{X}-2$. and let $H^{0}(X, \mathcal{L}(D))$ be projective. Then $\operatorname{deg} p^{*} D>$ $2 g_{\bar{X}}-2$ and $H^{0}(\bar{X}, \mathcal{L}(D))$ is projective. Thus $\bar{\pi}: \bar{X} \rightarrow \bar{Y}$ is weakly ramified and the congruence condition holds. But then $\pi$ is weakly ramified also, and the congruence condition holds for $D$, again by Lemma 1.5.

The following theorem generalizes Theorem 4.3 in [Kö2] and will be used in the formulation of the (main) Theorem 3.4. We refer the reader to page 1101 of the paper [Kö2] for an account of the nature, significance and history of the "ramification module" $N_{G, X}$ and for simplifications of formulae (1) and (2) when $\pi$ is tamely ramified.

Theorem 3.3. Let $\pi$ be weakly ramified. Then there is a projective $k[G]$-module $N_{G, X}$ such that

$$
\bigoplus^{n} N_{G, X} \cong \bigoplus_{P \in X} \bigoplus_{d=1}^{e_{P}^{t}-1} \bigoplus_{P}^{e_{P}^{w} \cdot d} \operatorname{Ind}_{I_{P}}^{G}\left(\operatorname{Cov}\left(\left(\mathfrak{m}_{P} / \mathfrak{m}_{P}^{2}\right)^{\otimes d}\right)\right),
$$

where Cov denotes the $k\left[I_{P}\right]$-projective cover. The class of $N_{G, X}$ in $K_{0}(G, k)$ is given by

$$
\left[N_{G, X}\right]=\left(1-g_{Y}\right)[k[G]]-\chi(G, X, \mathcal{L}(E))
$$

where $E$ denotes the $G$-equivariant divisor $E:=\sum_{P \in X}\left(e_{P}^{w}-1\right) \cdot P$.

Proof. Theorem 4.3 in [Kö2] yields that there is a projective $\bar{k}[G]$-module $N_{G, \bar{X}}$ such that

$$
\bigoplus^{n} N_{G, \bar{X}} \cong \bigoplus_{Q \in \bar{X}} \bigoplus_{d=1}^{e_{Q}^{t}-1} \bigoplus^{e_{Q}^{w} \cdot d} \operatorname{Ind}_{G_{Q}}^{G}\left(\operatorname{Cov}\left(\left(\mathfrak{m}_{Q} / \mathfrak{m}_{Q}^{2}\right)^{\otimes d}\right)\right),
$$

and that the class of $N_{G, \bar{X}}$ is given by

$$
\left[N_{G, \bar{X}}\right]=\left(1-g_{\bar{Y}}\right)[\bar{k}[G]]-\chi(G, X, \mathcal{L}(\bar{E}))
$$

where $\bar{E}:=\sum_{Q \in \bar{X}}\left(e_{Q}^{w}-1\right) \cdot Q=p^{*} E$. Thus $\left[N_{G, \bar{X}}\right]=\beta(\mathcal{C})$ where

$$
\mathcal{C}:=\left(1-g_{Y}\right)[k[G]]-\chi(G, X, \mathcal{L}(E)) \in K_{0}(G, k) .
$$

By Corollary 2.2, $\mathcal{C}$ is the class of some projective $k[G]$-module, say $N_{G, X}$. Using Lemma 1.3 and the injectivity of $\beta$, one easily shows that $N_{G, X}$ satisfies Formula (1).

For every point $P \in X$, let $f_{P}$ denote the residual degree $[k(P): k(\pi(P))]$. 
Theorem 3.4 (Equivariant Riemann-Roch formula). Let $\pi$ be weakly ramified.

(a) Let $P \in|X|$ be a closed point. For every $d \in\left\{0, \ldots, e_{P}^{t}-1\right\}$, there is a unique projective $k\left[G_{P}\right]$-module $W_{P, d}$ such that

$$
\operatorname{Ind}_{I_{P}}^{G_{P}}\left(\operatorname{Cov}\left(\left(\mathfrak{m}_{P} / \mathfrak{m}_{P}^{2}\right)^{\otimes(-d)}\right)\right) \cong \bigoplus^{f_{P}} W_{P, d}
$$

as $k\left[G_{P}\right]$-modules.

(b) Let $D=\sum_{P \in X} n_{P} \cdot P$ be a divisor on $X$ with $n_{P} \equiv-1 \bmod e_{P}^{w}$ for all $P \in X$. For any $P \in X$, we write

$$
n_{P}=\left(e_{P}^{w}-1\right)+\left(l_{P}+m_{P} e_{P}^{t}\right) e_{P}^{w}
$$

with $l_{P} \in\left\{0, \ldots, e_{P}^{t}-1\right\}$ and $m_{P} \in \mathbb{Z}$. Furthermore, for any $R \in Y$, fix a point $\tilde{R} \in \pi^{-1}(R)$. Then we have in $K_{0}(k[G])_{\mathbb{Q}}$ :

$$
\begin{aligned}
& \chi(G, X, \mathcal{L}(D)) \\
& \quad=-\left[N_{G, X}\right]+\sum_{R \in Y} \sum_{d=1}^{l_{\tilde{R}}}\left[\operatorname{Ind}_{G_{P}}^{G}\left(W_{P, d}\right)\right]+\left(1-g_{Y}+\sum_{R \in Y}[k(R): k] m_{\tilde{R}}\right)[k[G]] .
\end{aligned}
$$

Proof. We first show that under the preconditions of (b), the following holds in the Grothendieck group with rational coefficients $K_{0}(k[G])_{\mathbb{Q}}$ :

$$
\begin{aligned}
\chi(G, X, \mathcal{L}(D))=-\left[N_{G, X}\right]+\sum_{R \in Y} \frac{1}{f_{\tilde{R}}} \sum_{d=1}^{l_{\tilde{R}}}\left[\operatorname{Ind}_{I_{\tilde{R}}}^{G}\left(\operatorname{Cov}\left(\left(\mathfrak{m}_{\tilde{R}} / \mathfrak{m}_{\tilde{R}}^{2}\right)^{\otimes(-d)}\right)\right)\right] & \\
& +\left(1-g_{Y}+\sum_{R \in Y}[k(R): k] m_{\tilde{R}}\right)[k[G]]
\end{aligned}
$$

With suitably chosen divisors $D$, Formula (4) will then be used to show part (a). Formula (4) and part (a) obviously imply part (b).

For curves over algebraically closed fields, we have $f_{P}=1$ for all $P$, so Formula (4) coincides with Theorem 4.5 in [Kö2].

The injective homomorphism $\beta: K_{0}(G, k) \rightarrow K_{0}(G, \bar{k})$ maps $\chi(G, X, \mathcal{E})$ to $\chi\left(G, \bar{X}, p^{*} \mathcal{E}\right)$, and by Theorem 3.2, both of these Euler characteristics lie in the image of the respective Cartan homomorphisms. Hence it suffices to show that $\beta$ maps every summand of the right-hand side of formula (3) (applied to $X, D$ ) to the corresponding summand of the right-hand side applied to $\bar{X}, p^{*} D$.

From the proof of Theorem 3.3, we see that $\beta\left(\left[N_{G, X}\right]\right)=\left[N_{G, \bar{X}}\right]$.

By Lemma 1.5, we have $l_{Q}=l_{P}$ and $m_{Q}=m_{P}$ whenever $Q \in p^{-1}(P)$. Furthermore, the number of preimages of a point $R \in Y$ under $\pi: X \rightarrow Y$ is $\frac{n}{e_{\tilde{R}} f_{\tilde{R}}}$. For any $S \in|\bar{Y}|$, 
fix a point $\tilde{S} \in \bar{\pi}^{-1}(S)$. Using Lemma 1.3 , we see that

$$
\begin{aligned}
& \beta\left(\sum_{R \in Y} \frac{1}{f_{\tilde{R}}} \sum_{d=1}^{l_{\tilde{R}}}\left[\operatorname{Ind}_{I_{\tilde{R}}}^{G}\left(\operatorname{Cov}\left(\left(\mathfrak{m}_{\tilde{R}} / \mathfrak{m}_{\tilde{R}}^{2}\right)^{\otimes(-d)}\right)\right)\right]\right) \\
& =\sum_{Q \in \bar{X}} \frac{e_{Q}}{n} \sum_{d=1}^{l_{Q}}\left[\operatorname{Ind}_{G_{Q}}^{G}\left(\operatorname{Cov}\left(\left(\mathfrak{m}_{Q} / \mathfrak{m}_{Q}^{2}\right)^{\otimes(-d)}\right)\right)\right] \\
& =\sum_{S \in \bar{Y}} \sum_{d=1}^{l_{\tilde{S}}}\left[\operatorname{Ind}_{G_{\tilde{S}}}^{G}\left(\operatorname{Cov}\left(\left(\mathfrak{m}_{\tilde{S}} / \mathfrak{m}_{\tilde{S}}^{2}\right)^{\otimes(-d)}\right)\right)\right]
\end{aligned}
$$

Moreover, we have

$$
\beta\left(\left(1-g_{Y}+\sum_{R \in Y}[k(R): k] m_{\tilde{R}}\right)[k[G]]\right)=\left(1-g_{\bar{Y}}+\sum_{S \in \bar{Y}} m_{\tilde{S}}\right)[\bar{k}[G]]
$$

which completes the proof of Formula (4).

We now prove part (a). Let $P \in X$ be a closed point. For $d=0$, the statement is obvious because $\left(\mathfrak{m}_{P} / \mathfrak{m}_{P}^{2}\right)^{0}$ is the trivial one-dimensional $k(P)$-representation of $I_{P}$, so it decomposes into $f_{P}$ copies of the trivial one-dimensional $k(R)$-representation of $I_{P}$, where $R:=\pi(P)$. Hence we only need to do the inductive step from $d$ to $d+1$, for $d \in\left\{0, \ldots, e_{P}^{t}-2\right\}$.

If $\pi$ is unramified at $P$, then $e_{P}^{t}=1$, so there is no $d \in\left\{0, \ldots, e_{P}^{t}-2\right\}$. Hence we may assume that $\pi$ is ramified at $P$. Set $H:=G_{P}$, the decomposition group at $P$, and let $\pi^{\prime}$ denote the projection $X \rightarrow X / H=: Y^{\prime}$. For every closed point $Q \in|X|$ and for every $s \geq-1$, let $H_{Q, s}$ be the $s$-th ramification group at $Q$ with respect to that cover, as introduced in Section 1. Then we have $H_{Q, s}=G_{P} \cap G_{Q, s}$ for every $s \geq-1$ and every $Q \in|X|$. In particular, if $\pi$ is weakly ramified, then so is $\pi^{\prime}$. For $Q=P$, we get $H_{P, s}=G_{P, s}$ for all $s \geq-1$; in particular, the ramification indices and residual degrees of $\pi$ and $\pi^{\prime}$ at $P$ are equal.

Let now $D:=\sum_{Q \in|X|} n_{Q} \cdot Q$ be the $H$-equivariant divisor with coefficients

$$
n_{Q}=\left\{\begin{aligned}
(d+2) e_{Q}^{w}-1 & \text { if } Q=P \\
e_{Q}^{w}-1 & \text { otherwise }
\end{aligned}\right.
$$

Then formula (4) applied to $H, X, D$ gives

$$
\begin{aligned}
\chi(H, X, \mathcal{L}(D))=-\left[N_{H, X}\right] & +\frac{1}{f_{P}} \sum_{n=1}^{d}\left[\operatorname{Ind}_{I_{P}}^{H}\left(\operatorname{Cov}\left(\left(\mathfrak{m}_{P} / \mathfrak{m}_{P}^{2}\right)^{\otimes(-n)}\right)\right)\right] \\
& +\frac{1}{f_{P}}\left[\operatorname{Ind}_{I_{P}}^{H}\left(\operatorname{Cov}\left(\left(\mathfrak{m}_{P} / \mathfrak{m}_{P}^{2}\right)^{\otimes(-(d+1))}\right)\right)\right]+\left(1-g_{Y^{\prime}}\right)[k[H]]
\end{aligned}
$$

in $K_{0}(k[H])_{\mathbb{Q}}$. By the induction hypothesis, the sum from $n=1$ to $d$ in this formula is divisible by $f_{P}$ in $K_{0}(k[H])$; hence the remaining fractional term $\frac{1}{f_{P}}\left[\operatorname{Ind}_{I_{P}}^{H}\left(\operatorname{Cov}\left(\left(\mathfrak{m}_{P} / \mathfrak{m}_{P}^{2}\right)^{\otimes(-(d+1))}\right)\right)\right]$ must lie in $K_{0}(k[H])$. In other words, when writ$\operatorname{ing} \operatorname{Ind}_{I_{P}}^{H}\left(\operatorname{Cov}\left(\left(\mathfrak{m}_{P} / \mathfrak{m}_{P}^{2}\right)^{\otimes(-(d+1))}\right)\right.$ as a direct sum of indecomposable projective $k[H]$ modules, every summand occurs with a multiplicity divisible by $f_{P}$. This proves the assertion. 
In the proof of Theorem 3.4(a), we have used a preliminary version of the equivariant Riemann-Roch formula to show the divisibility of $\operatorname{Ind}_{I_{P}}^{G_{P}}\left(\operatorname{Cov}\left(\left(\mathfrak{m}_{P} / \mathfrak{m}_{P}^{2}\right)^{\otimes(-d)}\right)\right)$ by $f_{P}$, i.e. we have used a global argument to prove a local statement. This tells us very litte about the structure of the summands $W_{P, d}$, which leads to the question whether one could find a "local" proof for the divisibility. In two different situations, the following proposition provides such a proof, yielding a concrete description of $W_{P, d}$.

Proposition 3.5. Assume that $\pi$ is tamely ramified, let $P \in|X|$ and $d \in\left\{1, \ldots, e_{P}^{t}-1\right\}$.

(a) If $\operatorname{Gal}(k(P) / k(\pi(P)))$ is abelian, then we have $W_{P, d} \cong\left(\mathfrak{m}_{P} / \mathfrak{m}_{P}^{2}\right)^{\otimes(-d)}$ as $k\left[G_{P}\right]$ modules.

(b) If $I_{P}$ is central in $G_{P}$, then $W_{P, d}$ is of the form $W_{P, d}=\operatorname{Ind}_{I_{P}}^{G}\left(\chi_{d}\right)$ for some $k\left[I_{P}\right]$-module $\chi_{d}$. If moreover $G_{P} \cong I_{P} \times G_{P} / I_{P}$, then $W_{P, d} \cong\left(\mathfrak{m}_{P} / \mathfrak{m}_{P}^{2}\right)^{\otimes(-d)}$ as $k\left[G_{P}\right]$-modules.

Note that since every Galois extension of a finite field is cyclic, the first part of this proposition gives a "local" proof of Theorem 3.4(a) for the important case where $\pi$ is tamely ramified and the underlying field $k$ is finite.

Proposition 3.5 can be deduced from the following purely algebraic result. Note that, in this result, we don't use the notations introduced earlier in this paper; when Proposition 3.6 is being applied to prove Proposition 3.5, the fields $k$ and $l$ become the fields $k(\pi(P))$ and $k(P)$, respectively, the group $G$ becomes $G_{P}$ and $V$ becomes $\left(\mathfrak{m}_{P} / \mathfrak{m}_{P}^{2}\right)^{\otimes(-d)}$ which is viewed only as a representation of $I_{P}$ (and not of $G_{P}$ ) in Theorem 4.6(a).

Proposition 3.6. Let $l / k$ be a finite Galois extension of fields. Let $G$ be a finite group, and let $I$ be a cyclic normal subgroup of $G$, such that $G / I \cong \operatorname{Gal}(l / k)$, i.e. we have a short exact sequence

$$
1 \rightarrow I \rightarrow G \rightarrow \operatorname{Gal}(l / k) \rightarrow 1 .
$$

Let $V$ be a one-dimensional vector space over $l$ such that $G$ acts semilinearly on $V$, that is, for any $g \in G, \lambda \in l, v, w \in V$, we have $g \cdot(\lambda v+w)=\bar{g}(\lambda)(g \cdot v)+g . w$, where $\bar{g}$ denotes the image of $g$ in $\mathrm{Gal}(l / k)$.

(a) If $\operatorname{Gal}(l / k)$ is abelian, then we have $\operatorname{Ind}_{I}^{G} \operatorname{Res}_{I}^{G}(V) \cong \bigoplus^{(G: I)} V$ as $k[G]$-modules.

(b) If $I$ is central in $G$, then there is a (non-trivial) one-dimensional $k$-representation $\chi$ of $I$ such that $\operatorname{Res}_{I}^{G}(V) \cong \bigoplus^{(G: I)} \chi$ as $k[I]$-modules.

If moreover $G=I \times \operatorname{Gal}(l / k)$, then we have $\operatorname{Ind}_{I}^{G} \chi \cong V$ and $\operatorname{Ind}_{I}^{G} \operatorname{Res}_{I}^{G}(V) \cong$ $\bigoplus^{(G: I)} V$ as $k[G]$-modules.

Proof. (a) We have (isomorphisms of $k[G]$-modules):

$$
\begin{aligned}
\operatorname{Ind}_{I}^{G} & \operatorname{Res}_{I}^{G}(V) \\
& \cong V \otimes_{k} \operatorname{Ind}_{I}^{G}(k) \\
& \cong V \otimes_{k} k[G / I] \\
& \cong V \otimes_{k} k[\operatorname{Gal}(l / k)] \\
& \cong V \otimes_{k} l \\
& \cong \bigoplus_{\sigma \in \operatorname{Gal}(l / k)} V .
\end{aligned}
$$

by Corollary 10.20 in [CR] (cf. $\S 10 \mathrm{~A}$ in $[\mathrm{CR}]$ ) as $\operatorname{Gal}(l / k) \cong G / I$ 
The last two isomorphisms can be derived as follows. By the normal basis theorem, there is an element $x_{0} \in l$ such that $\left\{g\left(x_{0}\right) \mid g \in \operatorname{Gal}(l / k)\right\}$ is a basis of $l$ over $k$. The resulting isomorphism

$$
\begin{aligned}
k[\operatorname{Gal}(l / k)] & \rightarrow l \quad \text { given by } \\
{[g] } & \mapsto g\left(x_{0}\right) \quad \text { for every } g \in \operatorname{Gal}(l / k) .
\end{aligned}
$$

is obviously $k[G]$-linear. This is the second last isomorphism. For the last one, we define

$$
\begin{aligned}
\varphi: l \otimes_{k} V & \rightarrow \bigoplus_{\sigma \in \operatorname{Gal}(l / k)} V \text { by } \\
a \otimes v & \mapsto(\sigma(a) \cdot v)_{\sigma \in \operatorname{Gal}(l / k)} \quad \text { for every } a \in l, v \in V .
\end{aligned}
$$

$\varphi$ is an isomorphism of vector spaces over $k$, by the Galois Descent Lemma. If $\operatorname{Gal}(l / k)$ is commutative, then $\varphi$ is also compatible with the $G$-action on both sides: Let $a \in l, v \in V, g \in G$, then we have

$$
\begin{array}{r}
\varphi(g \cdot(a \otimes v))=\varphi(\bar{g}(a) \otimes g \cdot v)=((\sigma \bar{g})(a) \cdot g \cdot v)_{\sigma \in \operatorname{Gal}(l / k)}=((\bar{g} \sigma)(a) \cdot g \cdot v)_{\sigma \in \operatorname{Gal}(l / k)} \\
=g \cdot\left((\sigma(a) \cdot v)_{\sigma \in \operatorname{Gal}(l / k)}\right)=g \cdot \varphi(a \otimes v) .
\end{array}
$$

(b) Since $I$ is cyclic, it acts by multiplication with $e$-th roots of unity, where $e$ divides $|I|$. If $I$ is central in $G$, then it follows that the $e$-th roots of unity are contained in $k$. For if $h$ is a generator of $I$ and $h \cdot v=\zeta_{e} \cdot v$ for all $v \in V, \zeta_{e}$ an $e$-th root of unity, then we have for all $g \in G$ and all $v \in V$ :

$$
\bar{g}\left(\zeta_{e}\right)(g \cdot v)=g \cdot\left(\zeta_{e} v\right)=(g h) \cdot v=(h g) \cdot v=\zeta_{e}(g \cdot v) .
$$

Hence for everg $\bar{g} \in \operatorname{Gal}(l / k)$, we have $\bar{g}\left(\zeta_{e}\right)=\zeta_{e}$, which means that $\zeta_{e}$ lies in $k$. Let now $\left\{x_{1}, \ldots, x_{f}\right\}$ be a $k$-basis of $V$, where $f=(G: I)$. Then we have $V=k x_{0} \oplus \ldots \oplus k x_{f}$ not only as vector spaces over $k$, but also as $k[I]$-modules, since

$$
I x_{i}=\left\{\zeta_{e}^{j} x_{i} \mid j=0, \ldots, e-1\right\} \subseteq k x_{i}
$$

for every basis vector $x_{i}$. Furthermore, the summands $k x_{i}$ are isomorphic as $k[I]$ modules because $I$ acts on each of them by multiplication with the same roots of unity in $k$. Setting for example $k x_{1}=: \chi$, we can write

$$
\operatorname{Res}_{I}^{G}(V) \cong \bigoplus^{f} \chi
$$

as requested.

Assume now that $G=I \times \operatorname{Gal}(l / k)$. Then by the Galois Descent Lemma, we have

$$
V \cong l \otimes_{k} V^{\mathrm{Gal}(l / k)}
$$

as $k[G]$-modules, where $I$ acts trivially on $l$ and $\operatorname{Gal}(l / k)$ acts trivially on $V^{\mathrm{Gal}(l / k)}$. This is isomorphic to $l \otimes_{k} \chi$, where $\chi$ is regarded as a $k[G]$-module via the projection $G=I \times \operatorname{Gal}(l / k) \rightarrow I$. By the normal basis theorem, we have

$$
l \otimes_{k} \chi \cong \operatorname{Ind}_{I}^{G}(k) \otimes \chi=\operatorname{Ind}_{I}^{G}(\chi),
$$

so $V \cong \operatorname{Ind}_{I}^{G}(\chi)$ as requested. Together with what we have shown before, this implies the last identity of the proposition:

$$
\operatorname{Ind}_{I}^{G} \operatorname{Res}_{I}^{G}(V)=\operatorname{Ind}_{I}^{G}\left(\bigoplus^{f} \chi\right)=\bigoplus^{f} V .
$$




\section{Some variants of the main theorem}

Throughout the previous section, we have concentrated on the case where $\pi: X \rightarrow Y$ is weakly ramified and where the locally free $G$-sheaf we are considering comes from an equivariant divisor. If $\pi$ is tamely ramified, we have the following variant of Theorem 3.4 for locally free $G$-sheaves that need not come from a divisor. It generalizes Corollary $1.4(\mathrm{~b})$ in $[\mathrm{Kö} 1]$.

Theorem 4.1. Let $\pi: X \rightarrow Y$ be tamely ramified. Let $\mathcal{E}$ be a locally free $G$-sheaf of rank $r$ on $X$. For every closed point $P \in|X|$ and for $i=1, \ldots, r$, let the integers $l_{P, i} \in\left\{0, \ldots, e_{P}-1\right\}$ be defined by the following isomorphism of $k(P)\left[I_{P}\right]$-modules:

$$
\mathcal{E}(P) \cong \bigoplus_{i=1}^{r}\left(\mathfrak{m}_{P} / \mathfrak{m}_{P}^{2}\right)^{\otimes l_{P, i}}
$$

For every $R \in|Y|$, let $\tilde{R} \in|X|$ and $W_{\tilde{R}, d}$ be defined as in Theorem 3.4. Furthermore, let $N_{G, X}$ be the ramification module from Theorem 3.3. Then we have in $K_{0}(k[G])$ :

$$
\chi(G, X, \mathcal{E}) \equiv-r\left[N_{G, X}\right]+\sum_{R \in Y} \sum_{i=1}^{r} \sum_{d=1}^{l_{\tilde{R}, i}}\left[\operatorname{Ind}_{G_{\tilde{R}}}^{G}\left(W_{\tilde{R}, d}\right)\right] \quad \bmod \mathbb{Z}[G] .
$$

Moreover, one can show an equivariant Riemann-Roch formula for arbitrarily ramified covers $\pi: X \rightarrow Y$. Recall that in Theorem 3.2, we have shown that in virtually all cases where the Euler characteristic lies in the image of the Cartan homomorphism, the cover $\pi$ is weakly ramified. So in the general case, one cannot possibly find a formula in the Grothendieck group $K_{0}(k[G])$ of projective $k[G]$-modules. However, in the Grothendieck group $K_{0}(G, k)$ of all $k[G]$-modules, we have the following result, which generalizes Theorem 3.1 in [Kö2].

Theorem 4.2. Let $\mathcal{E}$ be a locally free $G$-sheaf. Then we have in $K_{0}(G, k)$ :

$$
n \chi(G, X, \mathcal{E})=C_{G, X, \mathcal{E}}[k[G]]-\sum_{P \in|X|} e_{P}^{w} \sum_{d=0}^{e_{P}^{t}-1} d\left[\operatorname{Ind}_{I_{P}}^{G}\left(\mathcal{E}(P) \otimes_{k(P)}\left(\mathfrak{m}_{P} / \mathfrak{m}_{P}^{2}\right)^{\otimes d}\right)\right]
$$

where

$$
C_{G, X, \mathcal{E}}=r\left(1-g_{X}\right)+\operatorname{deg} \mathcal{E}+\frac{r}{2} \sum_{P \in|X|}[k(P): k]\left(e_{P}^{t}-1\right) .
$$

We omit the proofs of Theorem 4.1 and Theorem 4.2 due to their similarity with the proof of Theorem 3.4.

\section{References}

[Bo] N. BorNe, Une formule de Riemann-Roch équivariante pour les courbes, thesis, Univ. Bordeaux, 1999. Available online at http://tel.archives-ouvertes.fr/tel-00001272/en

[CR] C.W. Curtis, I. ReineR, Methods of Representation Theory, Wiley, New York 1981 
[CW] C. Chevalley, A. WeIL, Über das Verhalten der Integrale erster Gattung bei Automorphismen des Funktionenkörpers, Hamb. Abh. 10 (1934), 358-361

[EL] G. Ellingsrud, K. Lønsted, An equivariant Lefschetz formula for finite reductive groups, Math. Ann. 251 (1980), 253-261

[Ha] R. Hartshorne, Algebraic Geometry, Springer, New York 1977

[Ka] E. KANI, The Galois-module structure of the space of holomorphic differentials of a curve, J. Reine Angew. Math. 367 (1986), 187-206

[Kö1] B. KöCK, Computing the equivariant Euler characteristic of Zariski and étale sheaves on curves, Homology Homotopy Appl. 7, No.3 (2005), 83-98

[Kö2] B. KöCK, Galois structure of Zariski cohomology for weakly ramified covers of curves, American Journal of Mathematics 126 (2004), 1085-1107

[Ku] E. Kunz, Kähler differentials, Vieweg, Wiesbaden 1986

[Na] S. NAKAJIMA, Galois module structure of cohomology groups for tamely ramified coverings of algebraic varieties, Journal of Number Theory 22 (1986), 115-123

[Se1] J.-P. SERre, Corps locaux, Publications de l'Institut de Mathématique de l'Université de Nancago VIII, Hermann, Paris 1962

[Se2] J.-P. SERRE, Représentations linéaires des groupes finis, Hermann, Paris 1978 